\section{P2-366 THE GLOBAL DISTRIBUTION OF DENGUE: PAST, PRESENT AND FUTURE IMPACTS OF CLIMATE CHANGE}

doi:10.1136/jech.2011.142976k.97

${ }^{1} \mathrm{E}$ van Kleef, ${ }^{2,3} \mathrm{H}$ Bambrick, ${ }^{*}{ }^{4} \mathrm{~S}$ Hales. ${ }^{1}$ Public Health \& Environment (PHE), WHO, Geneva, Switzerland; ${ }^{2}$ School of Medicine, University of Western Sydney, New South Wales, Australia; ${ }^{3}$ National Centre for Epidemiology and Population Health, The Australian National University, Canberra, Australia; ${ }^{4}$ Department of Public Health, University of Otago, Wellington, New Zealand

Introduction Approximately 2.5 billion people are at risk from dengue. Climate change could drive the epidemic potential of this disease through temperature impacts on both the virus and the mosquito vector. We describe the past and present global distribution of transmission and estimate possible changes to dengue distribution arising from climate change.

Methods Two systematic reviews:

1. Geographically defined case reports, and

2. Models of climate change impacts on transmission

Results The current distribution of dengue is generally less extensive than historical limits but several countries have recently reported transmission for the first time. Over the past century, dengue has contracted in the southern states of North America, much of Australia, parts of Southern Europe and Japan, China and South Africa, most likely due to piped water supplies and removal of water storage, changes in housing conditions and vector control measures. Modelling studies consistently project increased climatic suitability for transmission this century and an expansion of the geographic regions at risk of dengue. An increase in temperature of just $1^{\circ} \mathrm{C}$ can substantially increase transmission potential.

Conclusion Existing models of transmission provide incomplete projections of disease risk as the geographic limits to transmission result from a complex interaction between physical, ecological and social factors which have not been explicitly included in current, linear models. Urban poverty (eg, poor housing quality and stored domestic water) remains an important promoter of dengue transmission in regions with favourable climate, while factors associated with prosperity (eg, surveillance and control measures) can limit transmission in climate-suitable areas.

\section{P2-367 VARIATIONS IN PERINATAL DEPRESSION, ANXIETY AND SEVERE MENTAL ILLNESSES BY MATERNAL AGE AND SOCIOECONOMIC GROUP: A POPULATION-BASED STUDY IN UK PRIMARY CARE DATA}

doi:10.1136/jech.2011.142976k.98

L Ban, ${ }^{*}$ J E Gibson, J West, L J Tata. University of Nottingham, Nottingham, UK

Introduction Maternal perinatal mental illnesses are crucial public health issues and mostly addressed in UK primary care. This study was to assess whether there were variations in the burden of mental illnesses by age and socioeconomic status (SES).

Methods We randomly selected one pregnancy ending in a live birth for women age 15-45 years from 1994 to 2009 from The Health Improvement Network, a nationally representative electronic primary healthcare database. We assessed the association of maternal perinatal depression, anxiety and severe mental illnesses (eg, schizophrenia) with maternal age and SES (measured by the
Townsend Index) using logistic regression. We checked for evidence of effect modification between age and SES using a likelihood ratio test for interaction.

Results Compared with the highest socioeconomic group, women age $35-45$ years in the lowest socioeconomic group were 2.8 times (95\% CI 2.4 to 3.4) more likely to have depression during pregnancy and 2.4 times (95\% CI 2.1 to 2.7 ) after pregnancy whereas women age $15-24$ years were 1.4 times (95\% CI 1.1 to 1.8 ) and 1.6 times (95\% CI 1.4 to 1.8). Similar pattern was found for anxiety. After mutually adjusted for age and SES, older women or women with lower SES were also more likely to have severe mental illnesses.

Conclusion We find that women with lower SES are more likely to have perinatal mental illnesses. This is more evident in older than younger women for depression and anxiety. Perinatal healthcare professionals therefore should be aware of this and provide further effective interventions to this high-risk group.

\section{P2-368 WITHDRAWN}

P2-369 CORRELATION OF HOUSEHOLD SMOKING WITH INDIVIDUAL SMOKING, FYR OF MACEDONIA, 2009

doi:10.1136/jech.2011.142976k.100

${ }^{1} \mathrm{~A}$ Baraku, ${ }^{*} \mathrm{~A}$ Idrizaj, ${ }^{3} \mathrm{~B}$ Krasniqi, ${ }^{3} \mathrm{E}$ Baraku. ${ }^{1}$ lliria College, Institute for Scientific Research, Prishtina, Kosovo; ${ }^{2}$ General Hospital Rezonanca, Prishtina, Kosovo; ${ }^{3}$ liria College, Medical Sciences Faculty, Prishtina, Kosovo

Introduction Studies report various results on association of parental smoking with adolescent smoking initiation. This study researched smoking prevalence among students of the State University of Tetovo in comparison to household smoking; with the aim to establish the association for the given population and, if feasible, initiate subsequent intervention.

Methods Self-reported individual and family smoking was anonymously obtained from the first-year university students of Pharmacy and Dentistry, in year 2009. Prevalence of smoking was analysed in comparison to having smokers within their families, especially parent smokers and sibling smokers. Correlation between the modalities was tested with $\chi^{2}$ test and T-test of proportions. Validity scale was set for $\mathrm{p}<0.01$ and $\mathrm{p}<0.05$.

Results $26.7 \%$ of respondents (101) were smokers. Generally, the prevalence of smoking was significantly higher among students from smoking households $(37.9 \%)$ in comparison to students from non-smoking households (11.6\%), for $\mathrm{p}<0.01$. Similar behaviour was noticed when smoking prevalence was analysed in comparison to parent smokers $(40 \%$ vs $16.1 \%, \mathrm{p}<0.05)$ and sibling smokers (58.3\% vs $16.9 \%, \mathrm{p}<0.01)$. Smoking was prevailing among students whose mothers (66.7\%) respectively sisters (61.5\%) smoked, in comparison to those whose fathers (58.3) respectively brothers $(56.3 \%)$ smoked. Gender of smoker was significantly correlated to the gender of family smoker, for each $\mathrm{p}<0.01$.

Conclusion Within the study population, a strong correlation is found between household smoking-particularly maternal smoking-and individual smoking. There is a need of increasing parental especially mothers' awareness and responsibility about the impact of their smoking behaviour on adolescent smoking initiation 